\title{
BMJ Open Better Outcomes for Older people with Spinal Trouble (BOOST) Trial: a randomised controlled trial of a combined physical and psychological intervention for older adults with neurogenic claudication, a protocol
}

Esther Williamson, ${ }^{1}$ Lesley Ward, ${ }^{1}$ Karan Vadher, ${ }^{2}$ Susan J Dutton, ${ }^{2}$ Ben Parker, ${ }^{3}$ Stavros Petrou, ${ }^{3}$ Charles E Hutchinson, ${ }^{3}$ Richard Gagen, ${ }^{3}$ Nigel K Arden, ${ }^{4}$ Karen Barker, ${ }^{4,5}$ Graham Boniface, ${ }^{1}$ Julie Bruce, ${ }^{3}$ Gary Collins, ${ }^{2}$ Jeremy Fairbank, ${ }^{4}$ Judith Fitch, ${ }^{6}$ David P French, ${ }^{7}$ Angela Garrett, ${ }^{1}$ Varsha Gandhi, ${ }^{1}$ Frances Griffiths, ${ }^{3}$ Zara Hansen, ${ }^{1}$ Christian Mallen, ${ }^{8}$ Alana Morris, ${ }^{1}$ Sarah E Lamb ${ }^{1,2}$

To cite: Williamson $E$, Ward L, Vadher K, et al. Better Outcomes for Older people with Spinal Trouble (BO0ST) Trial: a randomised controlled trial of a combined physical and psychological intervention for older adults with neurogenic claudication, a protocol. BMJ Open 2018;8:e022205. doi:10.1136/ bmjopen-2018-022205

- Prepublication history for this paper is available online. To view these files, please visit the journal online (http://dx.doi org/10.1136/bmjopen-2018022205).

Received 6 February 2018 Revised 21 August 2018 Accepted 30 August 2018

A) Check for updates

(C) Author(s) (or their employer(s)) 2018. Re-use permitted under CC BY. Published by BMJ.

For numbered affiliations see end of article.

Correspondence to Dr Esther Williamson; esther.williamson@ndorms.ox. ac.uk

\section{ABSTRACT}

Introduction Neurogenic claudication due to spinal stenosis is common in older adults. The effectiveness of conservative interventions is not known. The aim of the study is to estimate the clinical and cost-effectiveness of a physiotherapist-delivered, combined physical and psychological intervention.

Methods and analysis This is a pragmatic, multicentred, randomised controlled trial. Participants are randomised to a combined physical and psychological intervention (Better Outcomes for Older people with Spinal Trouble (B00ST) programme) or best practice advice (control). Community-dwelling adults, 65 years and over, with neurogenic claudication are identified from community and secondary care services. Recruitment is supplemented using a primary care-based cohort. Participants are registered prospectively and randomised in a 2:1 ratio (intervention:control) using a web-based service to ensure allocation concealment. The target sample size is a minimum of 402 . The B00ST programme consists of an individual assessment and twelve 90 min classes, including education and discussion underpinned by cognitive behavioural techniques, exercises and walking circuit. During and after the classes, participants undertake home exercises and there are two support telephone calls to promote adherence with the exercises. Best practice advice is delivered in one to three individual sessions with a physiotherapist. The primary outcome is the 0swestry Disability Index at 12 months. Secondary outcomes include the 6 Minute Walk Test, Short Physical Performance Battery, Fear Avoidance Beliefs Questionnaire and Gait Self-Efficacy Scale. Outcomes are measured at 6 and 12 months by researchers who are masked to treatment allocation. The primary statistical analysis will be by 'intention to treat'. There is a parallel health economic evaluation and qualitative study.

Ethics and dissemination Ethical approval was given on 3 March 2016 (National Research Ethics Committee number:
Strengths and limitations of this study

The BOOST (Better Outcomes for Older people with Spinal Trouble) Trial is a large, multicentred, randomised controlled trial with a prespecified sample size estimate and includes health economic and qualitative evaluations.

- The primary outcome is the Oswestry Disability Index, but we also collect a range of secondary outcomes including objective physical capacity measures and self-reported pain, symptoms and mobility, which are highly relevant to this patient group.

- The intervention is individually tailored and uses group supervision to maximise the potential for cost-effectiveness.

- Due to the nature of the intervention, participants cannot be blinded to treatment allocation.

- At some sites, the same physiotherapist is delivering both trial interventions, but treatments are delivered according to a manualised protocol and quality control visits are conducted to reduce the risk of introducing bias to the trial.

16/L0/0349). This protocol adheres to the Standard Protocol Items: Recommendations for Interventional Trials checklist. The results will be reported at conferences and in peerreviewed publications using the Consolidated Standards of Reporting Trials guidelines. A plain English summary will be published on the BOOST website.

Trial registration number ISRCTN12698674; Pre-results.

\section{INTRODUCTION}

Neurogenic claudication (NC) is a condition that frequently affects older adults. ${ }^{1}$ The 
burden of symptoms is substantial. NC presents as pain, discomfort or other symptoms radiating from the spine into the buttocks and legs, which are provoked by walking or prolonged standing and relieved by sitting or lumbar flexion. ${ }^{2}$ Other signs and symptoms include weakness, altered sensation, fatigue and gait changes. ${ }^{2}$ Pain in the lower back is usual but not a necessary diagnostic feature. The symptoms of NC are thought to arise from pressure on the nerves and blood vessels in the spinal canal caused by degenerative changes narrowing the volume of the spinal canal. Narrowing may or may not be evident on radiological imaging. ${ }^{23}$ When narrowing is evident radiologically, the condition is termed lumbar spinal stenosis (LSS). The relationship between imaging results and symptoms is inconsistent as not all people with radiological narrowing report symptoms of $\mathrm{NC}^{23}$

Symptoms due to spinal stenosis are the most common reason for spinal surgery in people over 65 years of age. ${ }^{4}$ However, the effectiveness of surgery is unclear, and it exposes older people to considerable risk of complications, including wound infection and cardiorespiratory problems. ${ }^{4-6}$ Surgery is also expensive. Current clinical guidelines suggest that physiotherapy is an option for patients with symptoms arising from LSS before proceeding to surgery. ${ }^{7}$ However, we do not know whether physiotherapy is effective, nor which physiotherapy techniques should be used. ${ }^{8}$ A Cochrane systematic literature review reports that the current evidence for non-operative care for people with NC is very low to low quality. ${ }^{9}$ All recent reviews agree that higher quality trials are needed..$^{9-13}$ Despite NC being a condition associated with older age, interventions tested to date have not targeted age-associated changes in the musculoskeletal system of participants (such as generalised sarcopenia and frailty) or the psychological impact of pain. In order to generate high-quality evidence regarding non-surgical care for NC, our aim is to conduct a high-quality, multicentred, randomised controlled trial (RCT) of a physiotherapist-delivered, combined physical and psychological intervention.

\section{OBJECTIVES}

The following are the objectives:

- To estimate the clinical and cost-effectiveness of a physiotherapist-delivered, combined physical and psychological intervention for older adults with $\mathrm{NC}$ compared with best practice advice.

- To explore whether indicators of frailty, behavioural factors and radiological (MRI) biomarkers can identify groups of participants who are more likely to respond positively to the intervention using prespecified subgroup analyses.

- To conduct a parallel, longitudinal qualitative study with a sample of trial participants to better understand participant experiences of living and ageing with $\mathrm{NC}$, and to inform implementation if the intervention is successful.

\section{METHODS/DESIGN}

\section{Overview}

The study design is a multicentred RCT with embedded qualitative study and economic evaluation (see figure 1).

We are currently recruiting community-dwelling older adults with symptoms of NC. Recruitment opened on 25 July 2016 and we anticipate recruitment to be completed around June 2018. Participants are identified from National Health Service (NHS) physiotherapy and consultant spinal clinics in community and secondary care settings. In addition, participants are identified through a primary care-based cohort study (the Oxford Pain, Activity and Lifestyle (OPAL) cohort study). The OPAL cohort study is being conducted in the same localities as the trial.

The experimental intervention is a physiotherapist-delivered, combined physical and psychological programme. Participants attend an individual session, followed by 12 group sessions delivered over a 12-week period. During the individual session, participants undergo an assessment and are prescribed the exercises they will carry out during the group sessions tailored to their ability, symptom presentation and general health. The group sessions consist of (1) education and group discussion based on cognitive behavioural (CB) techniques; (2) warm-up and circuit exercises; and (3) a walking circuit. The education component focuses on pain management strategies, engagement with home exercises and increasing physical activity. The exercises target muscle strength, balance and flexibility, while the walking circuit aims to increase walking self-efficacy and mobility. The education component and supervised exercise are provided in groups of approximately six participants to maximise the potential for cost-effectiveness. There are two follow-up phone calls on completion of the group sessions to encourage adherence with the home exercise programme.

The comparator is advice given by a physiotherapist (best practice advice), ideally in one session, but up to two further review sessions are permissible. Advice includes self-management strategies, home exercises and encouragement to increase physical activity.

Participants are randomised in a 2:1 ratio (intervention:control) and followed up for 12 months (primary endpoint).

\section{Eligibility}

Participants are included in the trial if they fulfil the eligibility criteria listed in box 1 . In the UK, the majority of adults are registered with a primary care practice. Due to the pragmatic nature of this trial, we include people with symptoms consistent with the clinical presentation of $\mathrm{NC}$ rather than a diagnosis of spinal stenosis based on evidence of narrowing of the spinal canal on an MRI scan. $\mathrm{NC}$ presents as a cluster of symptoms easily recognised using simple self-report questions identified in a recent systematic literature review ${ }^{3}$ (table 1 ). These questions have excellent sensitivity and specificity for identifying $\mathrm{NC}^{3}$ and are used to screen for eligible participants. 


\section{Recruitment via OPAL cohort study}

Potential participants are identified from selfreport items in the baseline questionnaire of the OPAL cohort study, and sent an invitation

letter, response form and Participant Information Leaflet (PIL) for the BOOST RCT

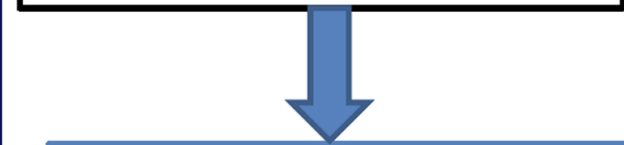

Potential participants are contacted by a researcher and invited for an eligibility assessment for the BOOST RCT

- Potential participants attend research assessment clinic.

- If eligible \& willing to take part, participants provide informed consent prior to randomisation to intervention or best practice advice ( $n=402$ minimum).

- Baseline data is collected.

- Participants are referred for MRI scan if they have not had one in the previous 12 months.

- Participants are invited to take part in the qualitative interview study (additional PIL). Those who provide separate consent for this will be subsequently contacted by the lead qualitative researcher.
The participant attends for treatment and is informed of treatment allocation by treating physiotherapist.

Follow-up at 6 months and 12 months: face-to-face appointment with a researcher.

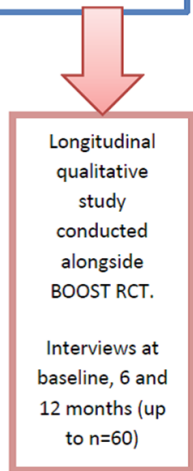

Figure 1 Study flow chart. BOOST, Better Outcomes for Older people with Spinal Trouble; NHS, National Health Service; OPAL, Oxford Pain, Activity and Lifestyle; RCT, randomised controlled trial.

The exclusion criteria are largely related to the participant being unable to participate in the intervention, for example, if they are unable to follow instructions or mobilise short distances without assistance. Participants are not excluded on the basis of any existing comorbidities unless their general practitioner (GP) feels inclusion in the study places them at risk.

\section{Approach}

Potential participants are approached via two routes:

Physiotherapy and consultant spinal clinics in community and secondary care NHS settings

Potential participants are identified by clinical staff in physiotherapy and consultant spinal clinics or from referrals. Staff are asked to identify potentially eligible patients based on age ( $\geq 65$ years) and symptoms (back and/or leg symptoms) and to screen out those on surgical waiting lists if that information is available. Clinical staff approach potentially eligible patients attending clinics, provide information about the study and ask if they are interested in being contacted by the research team. Clinical staff may also contact new referrals by telephone to inform them about the study. Potential participants who are interested and willing to be contacted by the research team are provided with a participant information leaflet (PIL), and their contact details are passed to the BOOST (Better Outcomes for Older people with Spinal Trouble) researcher for full eligibility screening. 


\section{Box 1 Eligibility criteria}

Inclusion criteria.

- Registered with a primary care practice.

- 65 years and over.

- Participant is willing and able to give informed consent for participation in the randomised controlled trial.

- Reports symptoms consistent with neurogenic claudication.

\section{Exclusion criteria.}

- Living in a residential care or nursing home.

- Has a terminal condition with a life expectancy of less than 6 months.

- Any substantial health or social concern that, in the opinion of the patient's general practitioner, would place the patient at increased risk or inability to participate, including known inability to provide informed consent, for example, dementia.

- Unable to walk $3 \mathrm{~m}$ (width of a small room) without the help of another person.

- On a surgical waiting list.

- Presents with cauda equina syndrome or signs of serious pathology requiring immediate referral for investigations.

- Cognitive impairment (defined as an Abbreviated Mental Test score of 6 or less).

- Registered blind.

- Unable to follow verbal instructions, which would make participation in the experimental treatment arm of the trial impractical, for reasons including severe hearing impairment not corrected by a hearing aid or inability to follow simple safety instructions (eg, English comprehension).

\section{The OPAL cohort study}

The OPAL cohort study is a population-based cohort study. Participants are identified from a random sample of patients aged over 65 years registered with each participating primary care practice. The OPAL cohort study is currently ongoing at 34 primary care practices, and will be described elsewhere.

Embedded within the cohort study postal questionnaires are self-report questions to identify individuals with possible NC (box 1). During the process of consent for the cohort study, OPAL participants are asked for additional consent for the University of Oxford to provide information and an invitation to clinical trials relevant to their clinical profile. OPAL participants who fulfil the initial criteria for the BOOST Trial (table 1) are invited to take part in eligibility screening for the trial and provided with the BOOST PIL. OPAL participants who accept the invitation for screening are then contacted by telephone for initial screening.

\section{Eligibility screening}

Potential participants identified via NHS spinal clinics or the OPAL cohort study are telephoned by the BOOST researcher (physiotherapists or research nurses) working at each site. During this telephone call, the researcher provides further information about the trial and completes initial eligibility checks. Eligible patients are invited to attend a research clinic appointment for a full assessment. If an individual requests to undertake the initial eligibility check in person, then this is arranged.

The research clinic appointment includes assessment of symptoms to ensure they are consistent with NC (using the questions in table 1), and screening for cauda equina syndrome or signs of serious pathology requiring immediate referral for investigations. Potential participants also undertake the Abbreviated Mental Test (AMT) ${ }^{14}$ to screen for cognitive impairment, which would make it difficult for a person to participate in the intervention. The AMT contains 10 items to assess orientation, registration, recall and concentration. This test can be used by any clinician and only takes $3-4$ minutes to administer. ${ }^{15}$ A score of 6 or below (out of 10 ) suggests cognitive impairment requiring further assessment and patients are advised to consult their GP. ${ }^{15-17} \mathrm{~A}$ mobility assessment is undertaken if required to ensure the participant is able to mobilise independently at least $3 \mathrm{~m}$ unassisted (with or without a walking aid).

\section{Informed consent}

On completion of the full eligibility assessment, eligible participants are asked to provide written informed consent prior to enrolling them into the trial. The consent is taken by a researcher who has completed training in the consent procedures for the BOOST Trial.

Table 1 Screening questions from the OPAL cohort study questionnaire ${ }^{3}$

\section{Questions}

Response required to be eligible for BOosT Trial

1. In the past 6 WEEKS, have you had back pain and/or pain or other symptoms Yes.

such as tingling, numbness or heaviness that travelled from your back into

your buttocks or legs?

(Note: If the answer to this question is no, then the participant will not complete the remaining questions).

1. Does standing make the pain or symptoms in your buttocks or legs worse? Yes to at least one of these questions.

2. Does walking make the pain or symptoms in your buttocks or legs worse?

3. Does sitting down make the pain or symptoms in your buttocks or legs better?

4. Does bending forward (eg, to push a shopping trolley) make the pain or symptoms in your buttocks or legs better? 


\section{Baseline assessment}

After providing consent, the participant then completes a baseline questionnaire and undergoes a clinical assessment by the researcher. Data collection is described in table 2. There are a number of variables only collected at baseline for the purposes of providing descriptive data on the sample. The participant is weighed using digital scales wearing light, indoor clothing with their shoes removed. Weight is recorded to the nearest $0.1 \mathrm{~kg}$. Height is measured using a stadiometer positioned against a wall. The participant stands on the platform, shoes removed, as upright as possible, hands by their side. The head plate of the stadiometer is lowered until it gently rests on the top of the participant's head and the height is recorded in metres from the measuring rod, to the nearest $0.001 \mathrm{~m}$ $(1 \mathrm{~mm})$.

The participant provides self-reported data including the demographic variables listed in table 2 , comorbidities including other pain problems (measured using the Nordic Pain Questionnaire ${ }^{18}$ ) and their current mobility status. Measures of mobility status include use of walking aids inside and outside, and self-rated walking speed. ${ }^{20}$ Change in mobility in the last year is measured using a 5-point scale constructed for the trial.

The STarT Back Screening Questionnaire ${ }^{21}$ is completed, allowing participants to be categorised according to their risk (low, medium or high) of developing persistent, disabling symptoms. ${ }^{21}$ Self-reported psychological factors with a potential impact on outcome are also collected. These include their confidence to exercise (Exercise Self-Efficacy Scale (short version) ${ }^{22}$ ) and their intention to carry out their home exercises using a question constructed for the trial (see table 2). Participants' attitudes to the physical changes associated with ageing are measured using the Attitudes to Aging Questionnaire-physical changes subscale. ${ }^{23}$

Variables collected at follow-up are described in the Outcome measures section.

\section{Imaging}

Alongside the RCT, there is an exploration of whether MRI scan parameters along with other baseline factors moderate response to physiotherapy treatment. Indirect visualisation by MRI is the gold standard for diagnosing LSS when a patient presents with NC, and is always undertaken before surgery, but not necessarily before conservative treatment. Increasingly, GPs have open access to MRI, and if MRI were predictive of response to conservative treatment this could aid GPs' clinical decision making. Despite the expense, there is remarkably little evidence about whether MRI scans can guide treatment choice effectively. Research evidence indicates that the fit between symptoms and MRI changes is poor. ${ }^{24}$ As MRI is currently the most common imaging investigation used, MRI data will be collected for all participants and we will systematically quantify the imaging characteristics. Pre-existing scans, taken in the 12 months preceding randomisation, will be used where possible to reduce the need for scanning.

Participants will be referred for an MRI study of the lumbar spine if they have not had one in the 12 months prior to randomisation. For these participants, the MRI scan will be taken after completion of other baseline data collection, and where possible before randomisation. Due to the nature of spinal stenosis, we would not expect spinal parameters to change markedly over a year-long period, hence the rationale for including existing scans. Practically, it is not possible to collect all MRI data prebaseline data collection as this may delay treatment and create unacceptable waiting times. For the subset of people who have MRI scans prior to randomisation, we will undertake formal subgroup analysis. We will explore other aspects of the relationship between functional outcomes and scan characteristics in additional analyses (not to be reported alongside the main trial results).

Consent for referral for a new MRI or use of an existing scan is obtained at the time of consent for the trial. Existing scans are transferred to a central repository for analysis in DICOM (Digital Imaging and Communications in Medicine) format.

The MRI data collection follows the protocol typical of NHS imaging departments. This is very similar across departments and efforts have been made to standardise the protocol where significant differences were identified.

We anticipate that a small number of participants will not have an MRI scan due to contraindications or by personal choice. Lack of an MRI scan does not exclude participants from the trial.

\section{Imaging protocol}

The MRI scan is performed supine, with the knees supported in flexion by a small foam wedge, resulting in relaxation of the normal lumbar lordosis. Imaging is performed using a dedicated spine phased array coil.

T1-weighted and T2-weighted sagittal imaging is followed by T2-weighted axial imaging of at least the lower three discs. The axial imaging is either taken as three separate blocks, each angulated and entered on the discs, or as a single block extending from L3 to S1.

Imaging parameters should be near those described in table 3. The BOOST Trial radiologist liaises with site to ensure data scans are suitable for data collection.

MRI scans are assessed by a single observer blinded to treatment allocation. Measurement of bony canal and dural sac cross-sectional area at each vertebral level allows assessment of central canal stenosis. The size of the lateral recess and neural exit foramen is measured and recorded quantitatively. The exact degree of narrowing to confirm stenosis is not well defined. In a review by Steurer $e t a l,{ }^{26}$ a dural sac cross-sectional area of less than $100 \mathrm{~mm}^{2}$ was considered diagnostic of central canal stenosis. Similarly, lateral recess depth and foraminal diameter measurements of less than $3 \mathrm{~mm}$ have been considered diagnostic of lateral recess and foraminal stenosis, respectively. 


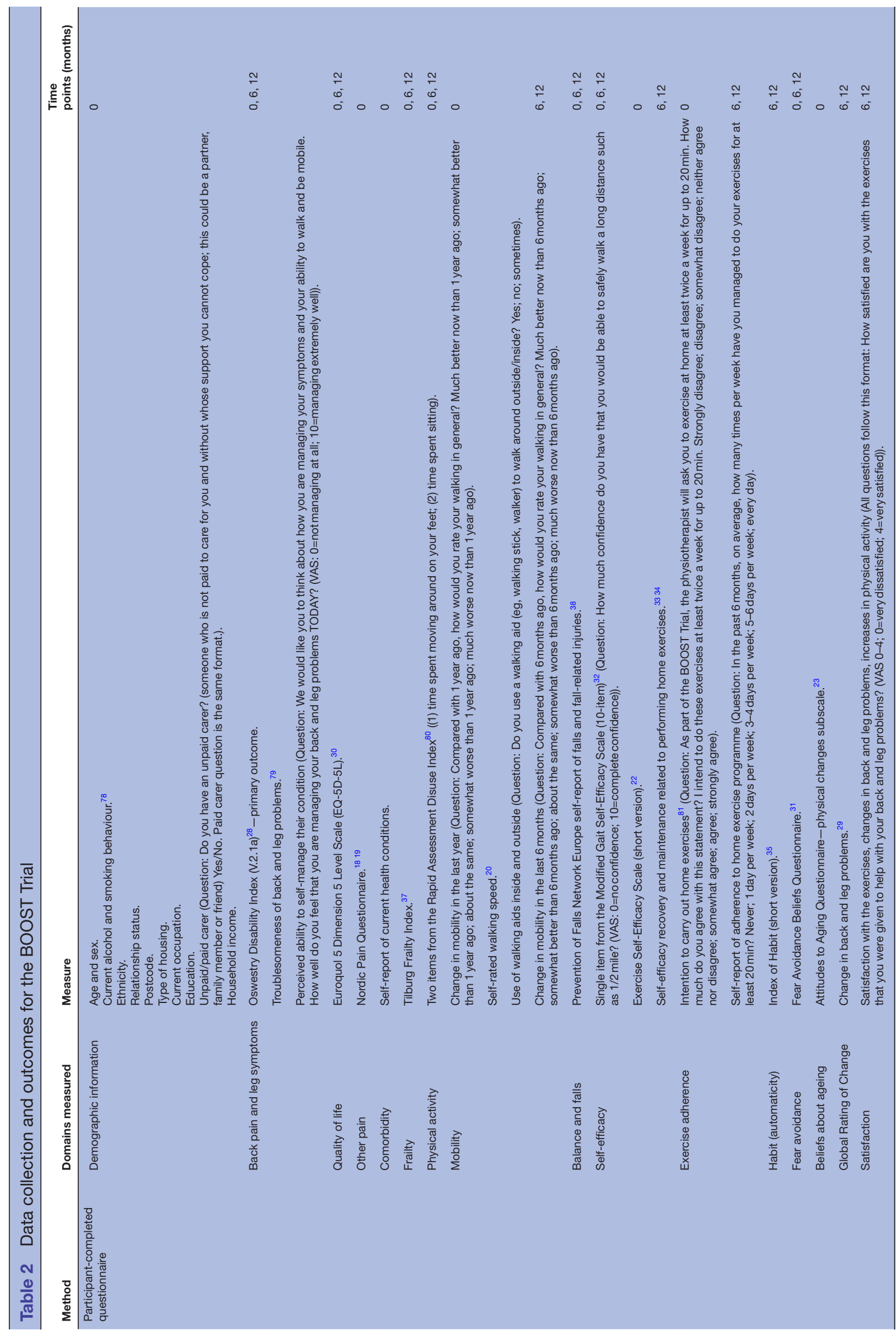

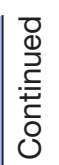




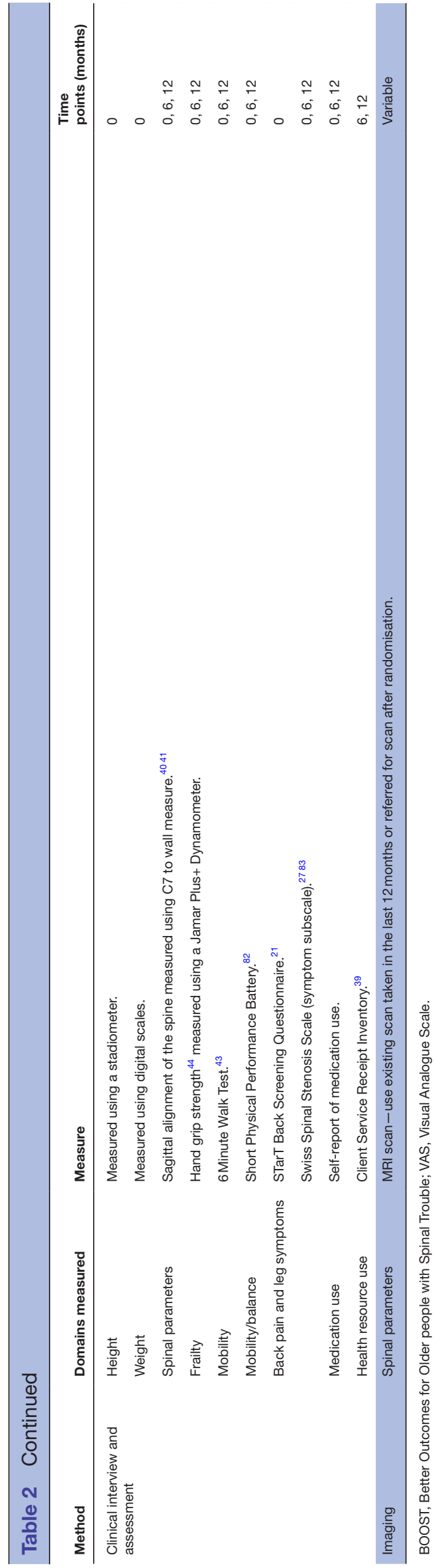

Provision of MRI results to participants

MRI scans requested for the purpose of the trial and not as part of a participant's clinical management are research investigations only. These are collected and assessed by the trial radiologist. If a serious spinal pathology is identified, the participant's GP and/or spinal consultant are immediately informed. If no serious pathology is identified, then scan results will be made available to participants at the end of the study if requested. MRI reports will be sent to each participant's GP or spinal consultant so that scan results are explained to the participant appropriately.

\section{Randomisation and masking}

Following baseline data collection, the researcher uses a web-based service to randomise the participants. During this process, the researcher is not informed of the treatment allocation. Instead, an automated email is sent directly to the physiotherapists who provide the interventions.

The web-based randomisation service is provided by the Oxford Clinical Trials Research Unit consistent with UK Clinical Research Collaboration (UKCRC) approved standard operating procedures, ensuring prospective registration and allocation concealment. Randomisation is stratified by centre, age (65-74 years and $75+$ years) and gender. Participants are randomised in a 2:1 ratio (intervention:control) to ensure that there are enough participants to run a group intervention and minimise waiting times.

Physiotherapists delivering the interventions and participants cannot be masked to treatment allocation. All participants receive an initial 1-hour appointment. For those randomised to the BOOST programme, this is an assessment prior to attending the group sessions. For those randomised to the control arm, it is their initial physiotherapy session to deliver best practice advice. During this appointment, participants are informed of their treatment allocation by the physiotherapist. To ensure that researchers collecting follow-up data remain masked to treatment allocation, physiotherapists and participants are asked not to share information about treatment allocation with researchers.

The trial statistician and the research staff undertaking quality assurance checks and the qualitative study are not blinded to treatment allocation. The remaining members of the trial management team, including all those who are involved in data management, are masked to treatment allocation.

\section{Outcome measures}

Follow-up data are collected at 6 and 12 months after randomisation, at a clinic appointment. The outcomes are listed in table 2.

\section{Primary outcome}

The primary outcome is low back pain disability measured using the Oswestry Disability Index (ODI V.2.1a) ${ }^{27} 28$ at 12 months after randomisation. The ODI is quite widely 
Table 3 Imaging parameters

\begin{tabular}{llllllllll}
\hline Sequ & FOV & Slice & Gap & TR & TE & ETL & Phase & Freq & Nex \\
\hline T2 sagittal & 370 & $13 / 4$ & 1 & 4061 & 102 & 23 & 320 & 512 & 3 \\
T1 sagittal & 370 & $13 / 4$ & 1 & 446 & 11 & 23 & 224 & 416 \\
T2 axial & 200 & $30 / 4$ & 1 & 4955 & 111 & 25 & 224 & 320 & 3 \\
\hline
\end{tabular}

ETL, echo train length; FOV, Field of view; Freq, Frequency; Nex, Number of excitations; Sequ, Sequence; TE, time to echo; TR, Time to repeat.

used as a measure for $\mathrm{NC}$ and very widely used in the field of back pain. A comparison of the psychometric properties of four of the most promising self-report measures for NC demonstrated that the ODI had superior properties to other measures. ${ }^{27}$ It is highly applicable to $\mathrm{NC}$ because it includes items on standing and walking. Scores range from 0 to 100 , with higher scores indicating greater disability. Participants are asked to consider back and leg symptoms when responding, including discomfort, heaviness, aching, tingling and numbness. Responses are not limited to the impact of back pain only.

\section{Secondary outcomes}

A range of self-reported and physical measures are collected to evaluate the impact of the intervention on key treatment targets (symptoms of NC, mobility, physical activity, strength, balance, frailty and falls, and cognitive and behavioural factors related to adherence with exercise and improving physical activity levels).

\section{Self-reported measures}

Self-reported measures related to symptoms and their impact include the Swiss Spinal Stenosis Questionnaire, ${ }^{27}$ Global Rating of Change, ${ }^{29}$ satisfaction with treatment using a 5-point scale constructed for the trial, health-related quality of life measured using the Euroquol 5 Dimension 5 Level Scale (EQ-5D-5L), ${ }^{30}$ and how well participants are managing their leg and back symptoms on a 10-point scale constructed for the trial.

Information is collected about cognitive and behavioural factors targeted during the intervention that are hypothesised to mediate the effects of the intervention. Fear avoidance is measured using the Fear Avoidance Beliefs Questionnaire. ${ }^{31}$ Self-efficacy is measured from different perspectives. Participants rate their confidence to walk half a mile using a single item from the Modified Gait Self-Efficacy Scale. ${ }^{32}$ To understand maintenance of exercise and physical activity, drawing on theoretical and empirical literature on this topic, ${ }^{33}$ participants also rate their confidence to restart their exercises having stopped them (self-efficacy recovery ${ }^{33}{ }^{34}$ ) and their confidence to maintain their exercises in the long term (self-efficacy maintenance ${ }^{33} 34$ ). Measures related to the adherence of exercises and increasing physical activity are collected using the Index of Habit, ${ }^{35}$ self-reported frequency of exercise and satisfaction with their attempts to increase their physical activity ${ }^{36}$ measured on a 5-point scale constructed for the trial. Change in mobility in the last
6 months is measured using a 5-point scale constructed for the trial.

A range of measures are collected to capture constructs related to ageing. Frailty is measured using the Tilburg Frailty Index, ${ }^{37}$ and information about falls and fall-related injuries is collected as recommended by the Prevention of Falls Network Europe. ${ }^{38}$ Health resource use will be collected using the Client Service Receipt Inventory. ${ }^{39}$

\section{Physical assessment}

A measure of postural alignment is undertaken to quantify the degree of thoracic kyphosis. ${ }^{40}$ The participant removes their shoes and socks and stands as upright as possible, with their sacrum and back against the wall, with hands by their sides. The researcher measures the distance from the spinous process of the seventh cervical vertebrae to the wall using a ruler. It is an alternative to the occiput to wall measurement, but reflects kyphosis better as it minimises error due to head position. 4041

We then collect measures related to mobility, balance and strength, which are important targets of the intervention and markers related to ageing and frailty.

The Short Physical Performance Battery (SPPB) ${ }^{42}$ measures three aspects of physical performance: standing balance, walking speed and the time taken to perform five chair stands. An overall score is given by adding the scores for each test. Researchers follow published guidance on the test which is, briefly, as follows ${ }^{42}$ :

\section{Standing balance}

Standing balance is rated on a scale of 0-4 according to the participant's ability to maintain three test positions (side-by-side stance, semitandem and full tandem) for $10 \mathrm{~s}$.

\section{Walking speed}

Walking speed is measured on an eight-feet long walking course with no obstructions for a further two feet at each end. The participant is instructed to 'Walk to the other end of the course at your usual speed, just as if you were walking down the street to go to the shop'. The time taken for the participant to walk between the two markers is recorded to the nearest $0.1 \mathrm{~s}$. The test is carried out twice and the faster of the two times is used to score the test on a scale of $0-4$. 


\section{Chair stands}

The participant sits in a straight-backed chair with their arms folded across their chest. They are given the following instructions: 'Now stand up straight 5 times in succession, as fast as you can'. The time taken to perform the five chair stands (from the initial sitting position to the final standing position at the end of the fifth stand) is used to score the test on a scale of $0-4$. If the participant is unable to complete the test, then they are given a score of 0 .

The 6 Minute Walk Test $(6 \mathrm{MWT})^{43}$ measures the distance that the participant is able to walk in 6 minutes. The researcher marks out an indoor walking course which is flat and straight and marked with cones at each end. The length of the test track is standardised at each site to ensure that the follow-up assessments are carried out on the same length test track. The recommended length of the course is a minimum of $10 \mathrm{~m}$ in total, but it is dependent on the space available at each site.

One lap consists of walking to the turnaround point of the course and returning to the start point. All researchers were provided with a $6 \mathrm{MWT}$ compact disc which is played during the test and counts down the 6 minutes of the walking test while the researcher counts the number of laps with a lap counter.

Prior to starting the test, the researcher also asks the participant if they have symptoms of NC. If they do not have any symptoms when starting, the participant is asked to verbally indicate if they begin to experience symptoms during the test. The distance at which their symptoms begin is recorded by the researcher.

The researcher measures the participant's hand grip strength $^{44}$ using a Jamar Plus+ Dynamometer and follows the protocol outlined by Roberts et $a l_{.}{ }^{45}$ The participant is seated in a chair with arms, with their hips, knees and ankles at $90^{\circ}$, and their feet flat on the ground. The participant's arm is supported on the armrest with their wrists level with the end of the armrest. During the test the researcher supports the weight of the dynamometer. Using standardised instructions, the participant is instructed to squeeze the handle of the dynamometer until they reach a maximal contraction and hold for 5 seconds. The procedure is repeated on the other side. Three measurements are taken on each hand allowing at least 30 seconds rest between measurements on the same hand. The highest reading is used as the summary measure.

\section{Follow-up procedures}

All participants are invited to attend a face-to face clinic appointment at 6 and 12 months. This is arranged by the researcher at each site. However, if a participant is unable to attend the clinic appointment, they are mailed a questionnaire that contains the primary outcome, all self-reported items and a participant completed version of the Client Service Receipt Inventory, but excludes the physical assessment. If the questionnaire is not returned within 2 weeks, then a second copy of the questionnaire is sent by the BOOST Trial Office as a reminder. If this is not returned within a further 2 weeks, then the BOOST Trial Office carries out a reminder phone call. After another 2 weeks, if the questionnaire has not been returned, then the BOOST Trial Office will attempt to contact the participant by telephone and collect core outcomes consisting of the primary outcome (ODI), pain troublesomeness rating, whether they are on a waiting list for spinal surgery, EQ-5D-5L, self-rated walking ability, falls and falls-related fractures, self-reported exercise adherence, and a brief version of the Client Service Receipt Inventory.

\section{Adverse events}

A safety reporting protocol has been developed to manage the reporting of related and unexpected serious adverse events (SAEs) and directly attributable adverse events (AEs). An AE is any untoward medical occurrence in a participant during a trial. There may or may not be a causal relationship with the trial intervention. AEs may be identified by the physiotherapists delivering the trial treatments or by researchers conducting follow-up assessments who have been trained in reporting procedures. SAEs must be reported to the trial management team within 24 hours of the physiotherapist or researcher becoming aware of the event. The Chief Investigator determines whether AEs require reporting to the Ethics Committee, Data Monitoring and Ethics Committee (DMEC) and Trial Sponsor.

\section{Training and quality assurance of the research protocol}

Researchers undergo approximately 4 hours of training covering eligibility screening, consent taking and data collection. They are provided with a manual containing detailed instructions for all trial procedures. All researchers undergo a quality assurance check to ensure they are following the trial protocol. This involves a BOOST team member observing the researcher carrying out the eligibility screening, taking consent and collecting trial data. Trial paperwork is checked for completeness. If any deviations from the protocol are identified, then further training is provided.

\section{Study interventions}

\section{Control intervention: best practice advice}

The control intervention is best practice advice which is delivered in a one-to-one session with a physiotherapist. Participants attend an initial appointment of up to 1 hour consisting of an assessment followed by the provision of advice and education. Advice and education includes education about NC, being physically active, use of medications, when to seek more advice and prescription of a home exercise programme (up to four exercises). Flexion and trunk stabilisations are recommended, but the physiotherapist may prescribe other exercises based on their assessment, if required. The physiotherapist may prescribe a walking aid if the assessment indicates (eg, to improve walking by increasing stability or for pain relief). Participants are provided with written information. 
Ideally, the control intervention should be delivered in one session. A maximum of two half-hour review appointments is permitted. During these sessions they can re-enforce verbal advice given, and review walking aids or exercises provided in the initial session, but are not permitted to provide treatments such as manual therapy, electrotherapy, acupuncture, hydrotherapy or structured exercise sessions.

The content of the control intervention has been informed by a survey of current physiotherapy practice $^{46}$ and through consultation with clinicians and patient representatives. Physiotherapy provision in the NHS is variable for this patient group. Many patients are not referred for physiotherapy, some receive advice on self-management at physiotherapy spinal clinics and some receive a course of physiotherapy comprising advice and exercises. Comer $e t a l^{8}$ compared a single advice and education session with up to six sessions of standard physiotherapy and showed no difference in outcomes. We recommend that the majority of participants receive one session of advice and education as no additional benefit has been demonstrated from extra sessions of standard physiotherapy. However, there are situations where the treating physiotherapist will feel that a review appointment is necessary (eg, if they have provided a walking aid and need to review its use) so this is permissible and we felt broadly reflected usual care in the NHS.

\section{Experimental intervention: the BOOST programme}

The BOOST programme will be described in full according to the Template for Intervention Description and Replication guidance ${ }^{47}$ elsewhere, including the rationale and development. A brief summary is provided here.

Participants are invited to attend twelve 90 minute group sessions over a 12-week period. We recommend that one physiotherapist delivers the BOOST programme to a group of six participants. If larger groups are conducted, then a physiotherapy assistant or another physiotherapist may be required. Prior to attending the programme, each participant attends an individual appointment (up to 1 hour) for an assessment and to set their individualised exercise and walking circuit targets for the group sessions. The baseline target for the strengthening exercise is tailored to each participant by varying the number of repetitions and sets, and the addition of weights as applicable.

Each session follows the same format. Participants take part in an education and discussion session, facilitated by the physiotherapist ( 30 minutes) and incorporating behavioural change strategies to encourage adherence with home exercises. This is followed by the exercise programme lasting approximately 1 hour. There is a short warm-up of seated exercises performed as a group, which includes arm raises, trunk rotation, pelvic tilting and knee lifts. Then participants undertake a circuit of strengthening (sitting knee extension, sit to stand, standing hip abduction and standing hip extension), stretching (a combined hip flexor and calf stretch) and a balance exercise. ${ }^{48}$ Each participant undertakes their individually tailored programme, which is progressed over the 12 weeks. The strengthening exercises are progressed by increasing the number of repetitions and sets, increasing the load or adding speed. The final part of the exercise element is a supervised walking circuit, designed to improve walking ability and fitness, ${ }^{48}$ which is also progressed over the 12 weeks by increasing the distance walked, increasing walking speed, adding balance challenges such as stairs or obstacles, or adding weights. The exercises carried out during the supervised sessions make up the home exercise programme (warm-up, exercise circuit and walking).

Participants attend the supervised sessions twice a week for sessions 1-6. As they progress through the programme, attendance becomes less frequent (weekly for sessions $7-9$, then fortnightly for sessions $10-12$ ). The home exercise programme is introduced during session 5 so that participants begin to undertake their home exercise programme while supported by the physiotherapist. On completion of the 12 group sessions, participants are asked to carry out their home exercise programme at least twice per week so that it becomes a habitual activity.

The physiotherapist monitors progress during the programme by asking participants to rate how well they feel they are managing their condition (0-10 Numerical Rating Scale) and how their symptoms are affecting walking (walking item from the ODI) at the pregroup assessment, and at sessions 3, 6, 9 and 12. At the end of the 12-week programme, the physiotherapist carries out two follow-up telephone reviews with each participant to promote long-term adherence with the home exercise programme. These take place approximately 1 and 2 months after completing the supervised sessions, and take approximately 15 minutes each.

\section{Concomitant care}

Participants may seek other forms of treatment during the trial if they feel it is necessary. Additional treatments accessed by participants, including contact with their GP or other health professionals, will be recorded on the Client Service Receipt Inventory ${ }^{39}$ at follow-up.

\section{Physiotherapist training and quality assurance of intervention delivery}

The interventions are delivered by physiotherapists registered with the Health and Care Professions Council. All physiotherapists delivering the BOOST programme attend a 1-day training course, are provided with an intervention manual and undertake 3 hours of online training. All physiotherapists delivering the control intervention attend 3 hours of training and are provided with an intervention manual. At some sites, the same physiotherapist delivers both arms of the trial. Both interventions are delivered according to a manualised protocol to reduce the risk of introducing bias to the study, and routine quality assurance checks are conducted. Visits are made 
to each site and at least one session of each intervention is observed. Feedback is provided to the physiotherapist on completion of the session and any issues or training needs identified. Another visit is arranged if substantial concerns are identified.

A structured checklist is used to monitor intervention delivery and ensure that all elements of the interventions are delivered as intended. We developed the education and discussion session of the BOOST programme with the assistance of a CB therapist who also assisted with training. The CB therapist helped to develop the checklist for the BOOST programme to ensure all the necessary components of the education and discussion section of the session were covered. The checklist is completed during the observed session. The education and discussion session may also be assessed via recording (depending on resources and BOOST staff capacity). All participants provide consent for sessions to be recorded for quality assurance purposes when they enrol in the study, and we seek verbal consent from the physiotherapist.

Following initial quality assurance checks and feedback, we also undertake fidelity assessments of both interventions that are not fed back to the physiotherapists. Feedback at this stage is not provided as we need to understand how this intervention would be delivered in the clinical setting if it were to be implemented.

A structured record of the interventions (treatment $\log$ ) is completed by the physiotherapists and used to monitor fidelity. We collect attendance rates to monitor adherence with the interventions. Additional site visits will be conducted if any problems with intervention delivery are identified.

\section{Sample size}

At $80 \%$ power and $5 \%$ two-sided significance levels, the proposed sample size is 321 participants in total providing data at 12-month follow-up (214 in the intervention arm and 107 in the control arm), after which inflation for potential loss to follow-up (20\%) yields an overall target of 402 (268 intervention, 134 control). If power is increased to $90 \%$, then a sample size of 429 (286 in the intervention arm and 143 in the control arm) is required, after which inflation for potential loss to follow-up (20\%) yields an overall target of 540 (360 intervention, 180 control).

These calculations have been based on the assumption that a between-group difference of five points is considered clinically significant on the ODI, with a baseline SD of 15 , consistent with published estimates in older populations and those with NC. ${ }^{50}$ This yields a standardised difference of 0.33 , a moderate effect size, which is consistent with being a reasonable target for a pragmatic trial. ${ }^{52}$

The loss to follow-up of $20 \%$ has been based on recent experiences of rehabilitation trials in older adults. ${ }^{53} \mathrm{We}$ estimate that the therapist effects will be negligible from data that we have generated/published from a series of trials using similar standardised interventions. Our recent trials of hand exercises in rheumatoid arthritis and $\mathrm{CB}$ interventions in low back pain generated an intracluster correlation (ICC) of less than $0.0001 .{ }^{54}{ }^{55}$ We anticipate about 20 therapists delivering the intervention, treating an average of 12-15 participants each. We have not incorporated a formal inflation for a therapist effect as the loss to follow-up allowance is generous and should mitigate against any moderate to large therapist effects.

The sample size is a minimum of 402 participants and a maximum of 540 , to be finalised following a review of the sample size assumptions (in particular any evidence of clustering or a larger baseline SD) by the DMEC. A number of assumptions in the sample size estimate will be checked at this interim time point and adaptations made if needed, including the baseline SD of the ODI and the observed ICC. The DMEC will review these assumptions at this time point and make recommendations regarding the final sample size to the Trial Management Group and Programme Steering Committee (PSC). No interim analysis of the primary outcome will be performed.

\section{Analysis}

Data will be reported in accordance with the Consolidated Standards of Reporting Trials guidelines for RCTs and the appropriate extensions. ${ }^{56} \mathrm{~A}$ final statistical analysis plan will be developed by the end of the recruitment period, and we provide an outline description here. The primary analysis will be 'intention to treat', where participants will be included in their randomised groups. Effect estimates together with their $95 \%$ CIs will be reported. The primary outcome, ODI at 12 months, will be analysed using a linear multivariable regression multilevel method to take account of any therapist effect and adjusted for the region, baseline ODI score, stratification and important prognostic variables.

Missing data will be minimised by careful data management and training. The nature and mechanism for missing variables and outcomes will be investigated, and if appropriate multiple imputation will be used. Sensitivity analyses will be undertaken, assessing the underlying missing data assumptions.

A secondary complier average causal effect (CACE) anal$\mathrm{ysis}^{57}$ will explore the effect of adherence with the intervention (attendance and the participants' engagement with the programme rated by the physiotherapist).$^{58}$ For the purposes of the primary CACE analysis, we will define adherence as attending at least 9 out of the 12 sessions $(75 \%)$. This would ensure that the majority of educational/discussion content is delivered. No one session is considered more important than another regarding educational/discussion content. Core CB concepts are introduced during the earlier sessions and are reiterated during subsequent sessions so attendance at nine sessions would ensure all core content is covered. Attendance at nine sessions will ensure that the participant is introduced to the home exercise programme and has undertaken the exercise programme for a minimum of 6 weeks (sessions 1-9 are delivered over a 6-week period). Six weeks of strength training has been shown to be sufficient to result 
in short-term improvements in muscle strength and physical function. ${ }^{59-61}$

We have also defined a priori subgroup criteria based on the published literature and will explore treatment effects by age (65-74 years/ $75+$ years), gender (male/female), Tilburg Frailty Index scores $(0-4 / 5+),{ }^{37}$ Fear Avoidance Beliefs Questionnaire scores $(0-14 / 15+),{ }^{62}$ STarT Back Risk Stratification score (low-risk/medium-risk/highrisk groups), ${ }^{21}$ hand grip strength (men: <30/30+; women $<20 / 20+)^{63}$ and SPPB scores (SPPB 0-6 low performance; SPPB 7-9 intermediate performance; SPPB 10-12 high performance $\left.{ }^{63}\right)$. Among participants who have an MRI scan prior to randomisation, we will estimate treatment effects in two subgroups defined by MRI parameters (cross-sectional spinal canal area cut-point of $100 \mathrm{~mm}^{2}$ ). ${ }^{26}$ Subgroup effects will be analysed using interaction with treatment tests and will be displayed using forest plots. ${ }^{64}$

We will carry out a series of additional exploratory subgroup and interaction analyses to identify other MRI scan parameters and baseline factors that predict change in ODI scores between baseline and 12 months. Interaction and polynomial terms will be considered when carrying out the exploratory analysis, and the analyses may be based on continuous or binary cut-points. These models will report variables that predict the outcome at 12 months with $95 \%$ CIs and $p$ value. These additional analyses will be presented in secondary publications and with appropriate caveats about the interpretation of exploratory subgroup analyses.

Further supplementary analysis may include mediation analysis to evaluate treatment mechanisms, and exploratory analyses of exercise dose effects including profiling of treatment response trajectories. These are a priori analyses based on the logic model used to develop the intervention, and will examine (1) whether the intervention affects the hypothesised mediators as intended, (2) whether changes in the hypothesised mediators relate to changes in outcomes, and (3) whether the effects of the intervention on the outcomes are attributable to changes in the hypothesised causal pathway.

\section{Economic evaluation}

A prospective economic evaluation, conducted from an NHS and personal social services perspective, is integrated into the trial design. The economic evaluation will estimate the difference in the cost of resources used by participants in the two arms of the trial, enabling costs and consequences to be compared between alternative forms of physiotherapy. The economic assessment method will adhere to the recommendations of the National Institute for Health and Care Excellence Reference Case. ${ }^{65}$

We will estimate the costs of delivering the intervention, including development and training, the cost of delivering sessions, and participant follow-up/management. Broader resource utilisation is captured through two principal sources: (1) participant interview administered at 6 and 12 months postrandomisation and (2) routine health service data collection systems (Hospital Episode
Statistics). Unit costs for health and social care resources will be derived from local and national sources. ${ }^{66}$ Costs will be standardised to current prices where possible. Health-related quality of life will be measured at baseline and at 6 and 12 months postrandomisation using the generic EuroQol EQ-5D-5L; national tariff sets will be used to generate quality-adjusted life-years (QALYs) ${ }^{67-69}$ We will in the first instance use self-report of the EuroQol EQ-5D-5L measure. Multiple imputation methods will be used to impute missing data and avoid biases associated with complete case analysis. ${ }^{70}$ The results of the economic evaluation will be expressed in terms of incremental cost per QALY gained. Non-parametric bootstrap estimation will be used to derive 95\% CIs for the mean cost and QALY differences between the trial groups, as well as to populate a cost-effectiveness plane. A series of sensitivity analyses will be undertaken to explore the implications of uncertainty on the incremental cost-effectiveness ratios and to consider the broader issue of the generalisability of the study results. The full details of the economic evaluation will be described in the health economic analysis plan.

\section{Qualitative study}

The aim of the qualitative study is to better understand participant experiences both of living and ageing with $\mathrm{NC}$, and their experience of the interventions delivered during the trial. Understanding the experiences of the participants will inform strategies for implementation if the intervention is clinically effective.

All participants recruited to the trial are eligible. As part of the consent process for the trial, participants are informed about the interview study and asked if they are willing to be contacted by a researcher to receive more information. Participants who agree are provided with an additional information sheet and contacted by the qualitative research team. Prior to starting the first interview, written consent is sought. Consent is reaffirmed verbally prior to each follow-up telephone interview.

We are interviewing participants at three time points over the course of the trial in order to capture physical, psychological, social and contextual changes. Topics explored include current impact of NC on day-to-day life and well-being, beliefs about the role of exercise in ameliorating symptoms, the role of exercise on slowing/ reversing physical decline, and how these beliefs impact on adherence to the treatments.

We estimate 60 participants will be required to ensure data saturation is reached in all three interviews while ensuring diversity of participants by age, gender, ethnicity and intervention arm, allowing for attrition over the course of the study. ${ }^{71}$ In any one recruitment site interview participants are recruited consecutively, and as recruitment proceeds sampling is adjusted to ensure diversity of age, gender, ethnicity and intervention arm.

Interviews are semistructured using prespecified openended questions. The interviewer uses prompts to further investigate responses and allows the participant to explore 
topics they feel are relevant. ${ }^{71}{ }^{73}$ The first interview takes place at a location convenient to the participant, usually their home. The second and third interviews are telephone interviews to reduce resource implications for the project. However, if a telephone conversation is unfeasible (eg, poor hearing), then subsequent interviews are conducted face to face.

The first interview takes place between randomisation and starting treatment. Participants do not yet know their treatment allocation. Questions focus on the impact of NC on the participant's physical and psychosocial health, their beliefs around exercise and ageing, and concerns and hopes regarding the intervention. The interview takes up to $90 \mathrm{~min}$ and is audio-recorded.

The second interview is approximately 1 month after completing treatment. Topics explored include the participant's experiences of the intervention, adherence to home exercises, and any changes in their symptoms, exercise and ageing beliefs, physical activity levels, or life circumstances. The third interview coincides with the 12-month follow-up assessment and further explores these topics, and how they may have changed after an extended period of self-management. Interview schedules are adapted to account for data captured in earlier interviews, and the interviewer has access to the outcome measures for each interviewee for exploration during the interview. The telephone interviews are recorded, and notes are transcribed by the interviewer from the audio recordings.

Audio recordings of first interviews are transcribed verbatim by an independent transcriber, anonymised and allocated an ID number. The telephone interview notes are checked against the audio recordings and linked to the first interview through the ID number. Participants are sent a copy of the transcripts if requested, and may delete any information they would not like to be used.

All transcripts are imported into NVivo and analysed using thematic analysis. ${ }^{74}$ Coding is undertaken as each transcript is received. We will undertake cross-case analysis. ${ }^{75}$ To understand trajectories of change in relation to back pain and NC, we will undertake longitudinal case comparative analysis, an approach to analysis used previously by the research team. ${ }^{76}$

\section{Trial management}

This trial is run by a UKCRC fully registered clinical trials unit, according to approved and audited standard operating procedures. All trial staff undergo regular training to ensure they are compliant with Good Clinical Practice and other relevant legislation and the requirements such as the Data Protection Act.

\section{Data management and checking}

All data are processed according to the Data Protection Act 1998 and all documents are stored safely in confidential conditions. Each participant is provided with a unique trial identification number. Data are entered manually onto the trial database (OpenClinica). The
BOOST Trial Office reviews all data collection forms for completeness and accuracy using automated validation checks, querying missing and nonsensical data with sites, according to trial-specific procedures which have been developed to ensure data quality.

\section{Patient and public involvement}

During the application process for this trial, we assembled a patient and public involvement (PPI) group and we have continued to work closely with them. JuF is the lead PPI representative and a coapplicant and contributed to the design of the trial. We appointed a PPI representative to be an independent member of the PSC. PPI engagement has been undertaken in face-to-face meetings and via emails and phone calls to make it as convenient as possible for the PPI group to contribute. PPI representatives have assisted with the development of the physiotherapy intervention. Two PPI representatives attended the intervention development day along with clinicians and researchers. One PPI representative carried out the proposed exercise programme in her home so she could give feedback on the practicalities of performing the proposed programme. PPI representatives helped us to develop the patient materials for the intervention.

PILs, consent forms and posters advertising the trial have been reviewed by the PPI group and they have provided feedback on the layout and wording. We have piloted questionnaires with our PPI group. PPI representatives have helped with developing interview schedules for the qualitative study, and we have carried out some practice interviews with the PPI representatives prior to undertaking the actual study.

\section{ETHICS AND DISSEMINATION}

Site-specific approvals were provided by the NHS Research and Development Departments at each participating site. The Chief Investigator will submit and, where necessary, obtain approval from the above parties for all substantial amendments to the original approved documents.

There were several ethical issues when designing this study. A study of older adults may identify individuals with previously unidentified cognitive impairment. As part of the screening process, participants complete the AMT. Individuals with a score of 6 or below (out of 10) are excluded as this suggests impaired cognitive function requiring further assessment. ${ }^{15-17}$ The researchers, conducting the eligibility screening, are trained to deal with this and to recommend that the person visit their GP to for further assessment.

The study screening procedures may identify individuals who have signs of serious spinal pathology (eg, cauda equina syndrome). In this case, the researcher would discuss it with the participant and as soon as possible with the local principal investigator and/or patient's spinal consultant or GP and take appropriate action.

Some participants will undergo an MRI scan as a research investigation. Some participants may want the 
results of their scan. However, as participants would not have had access to a scan as part of their routine NHS care, we will only make results available at the end of the trial unless we detect a serious spinal pathology (eg, spinal malignancy). In this situation, the participant's GP or spinal consultant will be informed immediately. If we were to provide the scan results, this may influence the beliefs of participants and their clinicians about the value of different treatments. This process is made clear to potential participants during the recruitment and consent procedures so participants understand the purpose of the MRI scan.

The PSC provides overall supervision of this research on behalf of the funder. It comprised the Chief Investigator, Project Lead, Trial Manager, Statistician and four independent members (including the committee chair). The PSC monitors trial progress and conduct and provides expert advice. In addition, a DMEC has been appointed. The DMEC consists of three independent experts with relevant clinical research and statistical experience. The DMEC has adopted the DAta MOnitoring Committees: Lessons, Ethics, Statistics (DAMOCLES) charter, ${ }^{77}$ which defines its terms of reference and operation in relation to oversight of the trial. No interim outcome analysis is planned. Direct access to research data will be granted to authorised representatives of the Sponsor (University of Oxford), regulatory authorities or the host institution for monitoring and/or auditing of the study to ensure compliance with regulations.

The results will be published in a peer-reviewed journal and at conferences, as well as in a report to the funder. A plain English summary will be made available on the BOOST website for participants (https://boost.octru.ox. ac.uk/).

\section{Author affiliations \\ ${ }^{1}$ Centre for Rehabilitation Research, Nuffield Department of Rhuematology, Orthopaedics and Musculskeletal Sciences, University of Oxford, Oxford, UK ${ }^{2} 0 x$ ford Clinical Trials Research Unit, Centre for Statistics in Medicine, Nuffield Department of Orthopaedics, Rheumatology and Musculoskeletal Sciences, University of Oxford, Oxford, UK \\ ${ }^{3}$ Warwick Medical School, University of Warwick, Coventry, UK \\ ${ }^{4}$ Nuffield Department of Orthopaedics, Rheumatology and Musculoskeletal \\ Sciences, University of Oxford, Oxford, UK \\ ${ }^{5}$ Oxford University Hospitals NHS Trust, Oxford, UK \\ ${ }^{6}$ Patient and Public Involvement Representative, Yorkshire, UK \\ ${ }^{7}$ Manchester Centre for Health Psychology, School of Health Sciences, University of Manchester, Manchester, UK \\ ${ }^{8}$ Arthritis Research UK Primary Care Centre, Institute for Primary Care and Health Sciences, Keele University, Keele, UK}

Acknowledgements Thank you to the patient and public involvement representatives who have provided advice and feedback on the development of the intervention, patient materials and conduct of the trial.

Contributors EW is the lead author of this manuscript. LW, SJD, KV, RG, SP and SEL were involved in writing the manuscript. All authors have read and approved the final manuscript. SEL is the Chief Investigator and the guarantor. EW, FG, SP, CEH, NKA, KB, JB, GC, JeF, JuF, DPF, ZH and CM are coapplicants on the grant awarded by the NIHR Programme Grants for Applied Research (reference: PTC-RP-PG-0213-20002) and were involved in the design of the study and its implementation, as were $A G$ as the trial manager, $A M$ as a research associate, SJD and $\mathrm{KV}$ as the trial statisticians, $\mathrm{GB}$ and VG as the research physiotherapists, LW as the postdoctoral researcher responsible for the qualitative study, $R G$ as the clinical research fellow (radiology) and BP as the trial health economist.

Funding This research is funded by the NIHR Programme Grants for Applied Research (reference: PTC-RP-PG-0213-20002). Preparatory work for the programme of research was supported by the Collaboration for Leadership in Applied Health Research and Care 0xford at Oxford Health NHS Foundation Trust. SEL and EW receive funding from the Collaboration for Leadership in Applied Health Research and Care 0xford at Oxford Health NHS Foundation Trust and are supported by the NIHR Biomedical Research Centre, Oxford. CM is funded by the NIHR Collaboration for Leadership in Applied Health Research and Care West Midlands, the NIHR School for Primary Care Research and an NIHR Research Professorship in General Practice (NIHR-RP-2014-04-026).

Disclaimer The views expressed are those of the author(s) and not necessarily those of the NHS, the NIHR or the Department of Health. The trial sponsor is the University of Oxford. The sponsor has no role in the trial design; collection, management, analysis or interpretation of data; writing of reports; and submission for publication.

Competing interests None declared.

Patient consent Not required.

Ethics approval Ethics approval for the BOOST Trial was given by the National Research Ethics Committee (REC number 16/L0/0349) on 3 March 2016.

Provenance and peer review Not commissioned; externally peer reviewed.

Open access This is an open access article distributed in accordance with the Creative Commons Attribution 4.0 Unported (CC BY 4.0) license, which permits others to copy, redistribute, remix, transform and build upon this work for any purpose, provided the original work is properly cited, a link to the licence is given, and indication of whether changes were made. See: https://creativecommons.org/ licenses/by/4.0/.

\section{REFERENCES}

1. Ishimoto $\mathrm{Y}$, Yoshimura N, Muraki S, et al. Prevalence of symptomatic lumbar spinal stenosis and its association with physical performance in a population-based cohort in Japan: the Wakayama Spine Study. Osteoarthritis Cartilage 2012;20:1103-8.

2. Suri P, Rainville J, Kalichman L, et al. Does this older adult with lower extremity pain have the clinical syndrome of lumbar spinal stenosis? JAMA 2010;304:2628-36.

3. de Schepper El, Overdevest GM, Suri P, et al. Diagnosis of lumbar spinal stenosis: an updated systematic review of the accuracy of diagnostic tests. Spine 2013;38:E469-E81.

4. Deyo RA, Mirza SK, Martin BI, et al. Trends, major medical complications, and charges associated with surgery for lumbar spinal stenosis in older adults. JAMA 2010;303:1259-65.

5. Kovacs FM, Urrútia G, Alarcón JD. Surgery versus conservative treatment for symptomatic lumbar spinal stenosis: a systematic review of randomized controlled trials. Spine 2011;36:E1335-51.

6. Zaina F, Tomkins-Lane C, Carragee E, et al. Surgical versus nonsurgical treatment for lumbar spinal stenosis. Cochrane Database Syst Rev 2016;1:Cd010264.

7. Kreiner DS, Shaffer WO, Baisden J, et al. Evidence-based clinical guidelines for multidisciplinary spine care: diagnosis and treatment of degenerative lumbar spinal stenosis: North American Spine Society, 2011.

8. Comer C, Redmond AC, Bird HA, et al. A home exercise programme is no more beneficial than advice and education for people with neurogenic claudication: results from a randomised controlled trial. PLoS One 2013;8:e72878.

9. Ammendolia C, Stuber KJ, Rok E, et al. Nonoperative treatment for lumbar spinal stenosis with neurogenic claudication. Cochrane Database Syst Rev 2013;8:CD010712.

10. May S, Comer C. Is surgery more effective than non-surgical treatment for spinal stenosis, and which non-surgical treatment is more effective? A systematic review. Physiotherapy 2013;99:12-20.

11. Jarrett MS, Orlando JF, Grimmer-Somers K. The effectiveness of land based exercise compared to decompressive surgery in the management of lumbar spinal-canal stenosis: a systematic review. BMC Musculoskelet Disord 2012;13:30.

12. Iversen MD, Choudhary VR, Patel SC. Therapeutic exercise and manual therapy for persons with lumbar spinal stenosis. Int J Clin Rheumtol 2010;5:425-37.

13. Macedo LG, Hum A, Kuleba L, et al. Physical therapy interventions for degenerative lumbar spinal stenosis: a systematic review. Phys Ther 2013;93:1646-60. 
14. Qureshi KN, Hodkinson HM. Evaluation of a ten-question mental test in the institutionalized elderly. Age Ageing 1974;3:152-7.

15. Sheehan B. Assessment scales in dementia. Ther Adv Neurol Disord 2012;5:349-58

16. Foroughan $\mathrm{M}$, Wahlund LO, Jafari Z, et al. Validity and reliability of Abbreviated Mental Test Score (AMTS) among older Iranian. Psychogeriatrics 2017:17:460-5.

17. Sarasqueta $C$, Bergareche A, Arce A, et al. The validity of Hodkinson's Abbreviated Mental Test for dementia screening in Guipuzcoa, Spain. Eur J Neurol 2001;8:435-40.

18. Kuorinka I, Jonsson B, Kilbom A, et al. Standardised Nordic questionnaires for the analysis of musculoskeletal symptoms. Appl Ergon 1987; 18:233-7.

19. Parsons S, Breen A, Foster NE, et al. Prevalence and comparative troublesomeness by age of musculoskeletal pain in different body locations. Fam Pract 2007;24:308-16.

20. Syddall HE, Westbury LD, Cooper C, et al. Self-reported walking speed: a useful marker of physical performance among communitydwelling older people? J Am Med Dir Assoc 2015;16:323-8.

21. Hill JC, Dunn KM, Lewis M, et al. A primary care back pain screening tool: identifying patient subgroups for initial treatment. Arthritis Rheum 2008;59:632-41.

22. Resnick B, Jenkins LS. Testing the reliability and validity of the selfefficacy for exercise scale. Nurs Res 2000;49:154-9.

23. Laidlaw K, Power MJ, Schmidt S. WHOQOL-OLD Group. The Attitudes to Ageing Questionnaire (AAQ): development and psychometric properties. Int J Geriatr Psychiatry 2007;22:367-79.

24. Moon ES, Kim HS, Park JO, et al. Comparison of the predictive value of myelography, computed tomography and MRI on the treadmill test in lumbar spinal stenosis. Yonsei Med J 2005:46:806-11.

25. Ishimoto Y, Yoshimura N, Muraki S, et al. Associations between radiographic lumbar spinal stenosis and clinical symptoms in the general population: the Wakayama Spine Study. Osteoarthritis Cartilage 2013;21:783-8.

26. Steurer J, Roner S, Gnannt R, et al. Quantitative radiologic criteria for the diagnosis of lumbar spinal stenosis: a systematic literature review. BMC Musculoskelet Disord 2011;12:175

27. Cleland JA, Whitman JM, Houser JL, et al. Psychometric properties of selected tests in patients with lumbar spinal stenosis. Spine $J$ 2012;12:921-31

28. Fairbank JC. Why are there different versions of the Oswestry Disability Index? J Neurosurg Spine 2014;20:83-6.

29. Kamper SJ, Maher CG, Mackay G. Global rating of change scales: a review of strengths and weaknesses and considerations for design. $J$ Man Manip Ther 2009;17:163-70.

30. Herdman M, Gudex C, Lloyd A, et al. Development and preliminary testing of the new five-level version of EQ-5D (EQ-5D-5L). Qual Life Res 2011;20:1727-36

31. Waddell G, Newton M, Henderson I, et al. A Fear-Avoidance Beliefs Questionnaire (FABQ) and the role of fear-avoidance beliefs in chronic low back pain and disability. Pain 1993;52:157-68.

32. Newell AM, VanSwearingen JM, Hile E, et al. The modified Gait Efficacy Scale: establishing the psychometric properties in older adults. Phys Ther 2012:92:318-28.

33. Kassavou A, Turner A, Hamborg T, et al. Predicting maintenance of attendance at walking groups: testing constructs from three leading maintenance theories. Health Psychol 2014:33:752-6.

34. Luszczynska A, Schwarzer R, Lippke S, et al. Self-efficacy as a moderator of the planning-behaviour relationship in interventions designed to promote physical activity. Psychol Health 2011;26:151-66.

35. Verplanken B, Orbell S. Reflections on past behavior: a self-report index of habit strength1. J Appl Soc Psychol 2003;33:1313-30.

36. Rothman AJ, Baldwin AS, Hertel AW, eds. Self-Regulation and behavior change: disentangling behavioral initiation and behavioral maintenance. New York: Guilford Press, 2011.

37. Gobbens RJ, van Assen MA, Luijkx KG, et al. The tilburg frailty indicator: psychometric properties. J Am Med Dir Assoc 2010;11:344-55.

38. Lamb SE, Jørstad-Stein EC, Hauer K, et al. Development of a common outcome data set for fall injury prevention trials: the Prevention of Falls Network Europe consensus. J Am Geriatr Soc 2005;53:1618-22.

39. Beecham J, Knapp M. Costing psychiatric interventions. London: Gaskell, 2001.

40. Wongsa S, Amatachaya P, Saengsuwan J, et al. Concurrent validity of occiput-wall distance to measure Kyphosis in communities. J Clin Trials 2012;02.

41. Viitanen JV, Heikkilä S, Kokko ML, et al. Clinical assessment of spinal mobility measurements in ankylosing spondylitis: a compact set for follow-up and trials? Clin Rheumatol 2000;19:131-7.
42. Guralnik JM, Simonsick EM, Ferrucci L, et al. A short physical performance battery assessing lower extremity function: association with self-reported disability and prediction of mortality and nursing home admission. J Gerontol 1994:49:M85-94.

43. Bennell K, Dobson F, Hinman R. Measures of physical performance assessments. Arthritis Care and Research 2011;63(S11):S350-S70.

44. Roberts HC, Syddall HE, Cooper C, et al. Is grip strength associated with length of stay in hospitalised older patients admitted for rehabilitation? Findings from the Southampton grip strength study. Age Ageing 2012;41:641-6.

45. Roberts HC, Denison HJ, Martin HJ, et al. A review of the measurement of grip strength in clinical and epidemiological studies: towards a standardised approach. Age Ageing 2011;40:423-9.

46. Comer CM, Redmond AC, Bird HA, et al. Assessment and management of neurogenic claudication associated with lumbar spinal stenosis in a UK primary care musculoskeletal service: a survey of current practice among physiotherapists. BMC Musculoskelet Disord 2009;10:121.

47. Hoffmann TC, Glasziou PP, Boutron I, et al. Better reporting of interventions: template for intervention description and replication (TIDieR) checklist and guide. BMJ 2014;348:g1687.

48. American College of Sports Medicine. Physical activity programs and behavior counseling in older adult populations. Med Sci Sports Exerc 2004;36:1997-2003.

49. Byrne C, Faure C, Keene DJ, et al. Ageing, muscle power and physical function: a systematic review and implications for pragmatic training interventions. Sports Med 2016:46:1311-32.

50. Whitman JM, Flynn TW, Childs JD, et al. A comparison between two physical therapy treatment programs for patients with lumbar spinal stenosis: a randomized clinical trial. Spine 2006;31:2541-9.

51. Pua YH, Cai CC, Lim KC. Treadmill walking with body weight support is no more effective than cycling when added to an exercise program for lumbar spinal stenosis: a randomised controlled trial. Aust $J$ Physiother 2007;53:83-9.

52. Altman D. Practical statistics for medical research. London: Chapman \& Hall/CRC, 1991.

53. Lamb SE, Williamson EM, Heine PJ, et al. Exercises to improve function of the rheumatoid hand (SARAH): a randomised controlled trial. Lancet 2015;385

54. Williams MA, Williamson EM, Heine PJ, et al. Strengthening And stretching for Rheumatoid Arthritis of the Hand (SARAH). A randomised controlled trial and economic evaluation. Health Technol Assess 2015;19:1-222.

55. Lamb SE, Lall R, Hansen Z, et al. A multicentred randomised controlled trial of a primary care-based cognitive behavioural programme for low back pain. The Back Skills Training (BeST) trial. Health Technol Assess 2010;14:1-253.

56. Schulz KF, Altman DG, Moher D, et al. CONSORT 2010 statement: updated guidelines for reporting parallel group randomised trials. BMC Med 2010;8:18.

57. Dunn G, Maracy M, Dowrick C, et al. Estimating psychological treatment effects from a randomised controlled trial with both noncompliance and loss to follow-up. Br J Psychiatry 2003;183:323-31.

58. Bellg AJ, Borrelli B, Resnick B, et al. Enhancing treatment fidelity in health behavior change studies: best practices and recommendations from the NIH Behavior Change Consortium. Health Psychol 2004;23:443-51.

59. Emerson NS, Stout JR, Fukuda DH, et al. Resistance training improves capacity to delay neuromuscular fatigue in older adults. Arch Gerontol Geriatr 2015;61:27-32.

60. Scanlon TC, Fragala MS, Stout JR, et al. Muscle architecture and strength: adaptations to short-term resistance training in older adults. Muscle Nerve 2014;49:584-92.

61. Fragala MS, Fukuda DH, Stout JR, et al. Muscle quality index improves with resistance exercise training in older adults. Exp Gerontol 2014;53:1-6.

62. Crombez G, Vlaeyen JW, Heuts PH, et al. Pain-related fear is more disabling than pain itself: evidence on the role of pain-related fear in chronic back pain disability. Pain 1999;80(1-2):329-39.

63. Cruz-Jentoft AJ, Baeyens JP, Bauer JM, et al. Sarcopenia: European consensus on definition and diagnosis: Report of the European Working Group on Sarcopenia in Older People. Age Ageing 2010;39:412-23

64. Brookes ST, Whitley E, Peters TJ, et al. Subgroup analyses in randomised controlled trials: quantifying the risks of false-positives and false-negatives. Health Technol Assess 2001;5:1-56.

65. NICE. Guide to the methods of technology appraisal. In: Excellence NIfHaC: NICE, 2013

66. Graves N, Walker D, Raine R, et al. Cost data for individual patients included in clinical studies: no amount of statistical analysis 
can compensate for inadequate costing methods. Health Econ 2002;11:735-9.

67. Dolan P. Modeling valuations for EuroQol health states. Med Care 1997;35:1095-108.

68. van Hout $B$, Janssen MF, Feng $Y S$, et al. Interim scoring for the EQ$5 \mathrm{D}-5 \mathrm{~L}$ : mapping the EQ-5D-5L to EQ-5D-3L value sets. Value Health 2012;15:708-15.

69. Brazier J, Roberts J, Deverill M. The estimation of a preferencebased measure of health from the SF-36. J Health Econ 2002;21:271-92.

70. Sterne JA, White IR, Carlin JB, et al. Multiple imputation for missing data in epidemiological and clinical research: potential and pitfalls. BMJ 2009;338:b2393.

71. Britten N. Qualitative interviews in medical research. BMJ 1995:311:251-3.

72. Morse JM. Determining sample size. Qual Health Res 2000;10:3-5.

73. Cohen D, Crabtree B. Semi-structured Interviews. Qualitative research guidelines project. 2006.

74. Braun V, Clarke V. Using thematic analysis in psychology. Qual Res Psychol 2006;3:77-101.

75. Miles M, Huberman A. Qualitative data analysis. Second edn. Thousand Oaks: SAGE Publications Ltd, 1994.

76. Griffiths F, Borkan J, Byrne D, et al. Time, complexity and chronic back pain: a longitudinal qualitative study: North America Primary Care Research Group, 2009.
77. DAMOCLES Study Group, NHS Health Technology Assessment Programme. A proposed charter for clinical trial data monitoring committees: helping them to do their job well. Lancet 2005;365:711-22.

78. Guralnik JM, Fried LP, Simonsick EM, et al. The Women's Health and aging study: health and social characteristics of older women with disability. Bethesda, MD: National Institute of Aging, 1995.

79. Parsons S, Carnes D, Pincus T, et al. Measuring troublesomeness of chronic pain by location. BMC Musculoskelet Disord 2006;7:34.

80. Shuval K, Kohl HW, Bernstein I, et al. Sedentary behaviour and physical inactivity assessment in primary care: the Rapid Assessment Disuse Index (RADI) study. Br J Sports Med 2014;48:250-5.

81. Fishbein M, Ajzen I. Predicting and changing behavior: the reasoned action approach. New York: Psychology Press, 2010.

82. Guralnik JM, Ferrucci L, Pieper CF, et al. Lower extremity function and subsequent disability: consistency across studies, predictive models, and value of gait speed alone compared with the short physical performance battery. J Gerontol A Biol Sci Med Sci 2000;55:M221-31.

83. Pratt RK, Fairbank JC, Virr A. The reliability of the shuttle walking test, the swiss spinal stenosis questionnaire, the oxford spinal stenosis score, and the oswestry disability index in the assessment of patients with lumbar spinal stenosis. Spine 2002;27:84-91. 